\title{
Serum Cystatin C Level for Evaluation of Renal Functions in Women with Severe Preeclamptic Toxemia
}

\author{
MOHAMED R. MONTASER, M.Sc.*; AHMED M. OSMAN, M.D.*; WESSAM S. MOHAMED, M.D.** and \\ AHMED H. ABOU FREIKHA, M.D.* \\ The Departments of Obstetrics \& Gynecology* and Clinical Pathology**, Faculty of Medicine, Tanta University
}

\begin{abstract}
Background: Preeclampsia is a complex and serious multisystem disorder of pregnancy with a worldwide incidence of $5-7 \%$ and contributes significantly to maternal and perinatal morbidity and mortality. Altered renal function is an essential component of the pathophysiological process in PE, so close monitoring of renal function is important to ensure a timely delivery before serious renal damage occurs.
\end{abstract}

Aim of the Work: To evaluate diagnostic value of cystatin $\mathrm{C}$ serum levels as an alternative marker of renal function in women with severe preeclamptic toxemia versus matching cases of normal pregnancy and compare it with the traditional markers of renal function, Creatinine, urea and serum uric acid.

Patients and Methods: A case-control study includes 80 Primigravidae with singleton pregnancy and gestation $\geq 28$ weeks and were divided into two groups, Group 1: (40 patients) with severe preeclampsia and Group 2: (40 patients) as a control group (normotensive and nonproteinuric). Markers of kidney function were investigated in two groups of pregnant women and comparison between both groups as regards to cystatin $\mathrm{C}$ serum levels, creatinine, blood urea and serum uric acid was done.

Results: The difference in the mean serum uric acid level $(5.10 \pm 0.96$ versus $4.70 \pm 0.63 \mathrm{mg} / \mathrm{dl})$ was significantly higher in preeclampsia $(p=0.027)$, the specificity was $100 \%$ and sensitivity of the test was $87.5 \%$, the difference in the mean serum concentration of creatinine $(0.94 \pm 0.16$ versus $0.86 \pm$ $0.12 \mathrm{mg} / \mathrm{dl})$ was significantly higher in preeclampsia $(p=0.028)$, the specificity of the test was $100 \%$ and the sensitivity was $82.5 \%$. Serum cystatin $C$ level has a mean $(1.26 \pm 0.21$ versus $0.75 \pm 0.05 \mathrm{mg} / \mathrm{l})$ which was significantly higher in preeclampsia $(p=0.001)$ with a specificity $100 \%$ and sensitivity $96 \%$.

Conclusion: It is concluded that among serum creatinine, blood urea, serum uric acid \& serum cystatin C, Serum cystatin $\mathrm{C}$ is the earliest \& a better marker of renal dysfunction in preeclampsia.

Key Words: Cystatin C-Renal function - Preeclampsia.

Correspondence to: Dr. Mohamed R. Montaser, The Department of Obstetrics \& Gynecology Faculty of Medicine, Tanta University

\section{Introduction}

PREECLAMPSIA is a disorder of widespread vascular endothelial malfunction with vasospasm which occurs after 20 weeks' gestation and can present up to 4-6 weeks' post-partum. It is clinically defined by hypertension and proteinuria, with or without pathologic edema. PE is subdivided into mild and severe, with severe form showing more prominent signs and symptoms of end-organ damage that may end in life-threatening disease. PE can cause renal impairment, hepatic dysfunction, jaundice, abdominal pain, disseminated intravascular coagulation, convulsions or normotensive proteinuria. This variability was explained as the sum of consequences of diffuse endothelial dysfunctio [1]

The renal dysfunction can be due to disruption of the glomerular filtration barrier through glomerular capillary endothelial cell injury, podocytes are also disrupted. This leads to proteinuria and hypertension. Arteriolar endothelial injury also occurs in PE and may induce significant narrowing of arteriolar Lumina. So glomerular filtration is compromised and this results in glomerular hypoperfusion and diminished glomerular filtration rate with renal compensation in the form of elevated blood pressure [2] .

In the last 40 years, serum creatinine $(\mathrm{SCr})$ has become the most commonly used serum marker of renal function. Its rate of appearance in the bloodstream is related to muscle mass and is affected by age and gender [3]. SCr circulates in the bloodstream unbound to any plasma proteins and is freely filtered by the glomerulus. It is not reabsorbed by the proximal tubules, but is secreted in small amounts, that are subject to intra- and inter- 
individual variation [4]. As its plasma concentrations increase, tubular secretion of SCr increases, leading to an overestimation of GFR in patients with moderate to severe decreases in GFR $(<50 \mathrm{~mL} / \mathrm{min})$ [5]

Cystatin C is a 122-amino acid, 13 kilo Dalton protein which is a member of the family of cysteine proteinase inhibitors. It is the product of a "housekeeping" gene expressed in all nucleated cells and is produced at a constant rate [6]. Because of its small size and basic pI ( 9.0), CysC is freely filtered by the glomerulus. It is not secreted, but it is reabsorbed by tubular epithelial cells and subsequently catabolized so that it does not return to the blood flow [7]. CysC is almost an ideal marker of GFR because of its features such as: easy passage through the glomerular filter, absent tubular secretion and absent extra renal excretion. A significant number of studies indicate that cysC is superior to $\mathrm{SCr}$ as a marker of the GFR [8], especially in the early stages of chronic kidney disease (CKD), i.e. in the creatinine "blind" area (in GFR between $60-90 \mathrm{ml} / \mathrm{min} / 1.73 \mathrm{~m}^{2}$ ) [9]. Reference values differ in many populations and with sex and age. Across different studies, the mean reference interval was between 0.52 and $0.98 \mathrm{mg} / \mathrm{L}$ [10]

The level of cysC during the first and second trimester of pregnancy does not differ significantly from its level in non-pregnant women. In the third trimester, an increase occurs, up to $29-39 \%$ when compared to the previous two trimesters [11]. A significant increase in the serum level of cysC in pregnancy can be found in PE. Moreover, its level is not only significantly higher in the pregnant women who develop PE when compared to women with normal pregnancies, but the increased levels of serum cysC are also present a few weeks or months before the development of PE. This increase is due to impaired renal function, and increased placental production of cysC [12].

\section{Aim of the work:}

To evaluate diagnostic value of cystatin $\mathrm{C}$ serum levels as an alternative marker of renal function in women with severe preeclamptic toxemia versus matching cases of normal pregnancy and compare it with the traditional markers of renal function, Creatinine, urea and serum uric acid.

\section{Patients and Methods:}

This study involved 40 pregnant women with severe preeclampsia and 40 women with normal pregnancy (control group) in the 3 rd trimester attending Department of Obstetrics and Gynecology, at Tanta University Hospital from April 2016 to April 2017. All patients were subjected to thorough history taking with special emphasis on Gestational age (in weeks), History of medical disease (as chronic hypertension, renal disease, diabetes mellitus, thyroid disorders, liver diseases) and Clinical examination regarding Blood pressure and Body mass index. Obstetrical abdominal ultrasound was done by Fukuda Denshi Uf-850XTD for Gestational age (BPD, AC, FL), Amniotic fluid, Viability and Doppler study of umbilical, middle cerebral and uterine arteries. Routine investigations as Fasting blood sugar, Liver enzymes, complete blood count, urine analysis, serum urea, Uric acid, Creatinine and Estimation of serum cystatin were done. After that statistical analysis of the data was done by IBM computer using SPSS (statistical program for social science) version 20 as follows: Description of quantitative variables as range; mean and SD; description of qualitative variables as number and percentage. ANOVA test (Analysis of Variance) was used to compare quantitative variables between groups; Chi-square test was used to compare qualitative variables between groups. Receiver operator characteristic curve (ROC) was used to find out sensitivity, specificity, +ve predictive value $(+\mathrm{PV}),-$ ve predictive value $(-\mathrm{PV})$ and accuracy.

\section{Results}

Markers of kidney function were investigated in the two groups. All renal parameters including cystatin $\mathrm{C}$ were significantly raised in $\mathrm{PE}$ group compared with control group. The difference in the mean serum uric acid level $(5.10 \pm 0.96$ versus $4.70 \pm 0.63 \mathrm{mg} / \mathrm{dl})$ was significantly higher in the preeclampsia ( $p=0.027$ ), the difference in the mean serum concentration of creatinine $(0.94 \pm 0.16$ versus $0.86 \pm 0.12 \mathrm{mg} / \mathrm{dl})$ was significantly higher in preeclampsia $(p=0.028)$, the difference in the mean concentration of blood urea $(25.55 \pm 5.71$ versus $22.85 \pm 5.39 \mathrm{mg} / \mathrm{dl})$ was significantly higher in preeclampsia ( $p=0.033)$. Serum cystatin $C$ level has a mean $(1.26 \pm 0.21$ versus $0.75 \pm 0.05 \mathrm{mg} / \mathrm{l})$ which was significantly higher in preeclampsia $(p=0.001)$.

AUC for serum uric acid level was 0.910, the specificity was $100 \%$ and sensitivity of the test was $87.5 \%$, with accuracy $94 \%$. AUC for serum creatinine level was 0.972 , the specificity was $100 \%$ and sensitivity of the test was $85 \%$, with accuracy $91 \%$. AUC for serum cystatin C level was 0.814 , the specificity was $100 \%$ and sensitivity of the test was $96 \%$, with accuracy $97.5 \%$. 
Table (1): Comparison between the two studied groups according to renal function.

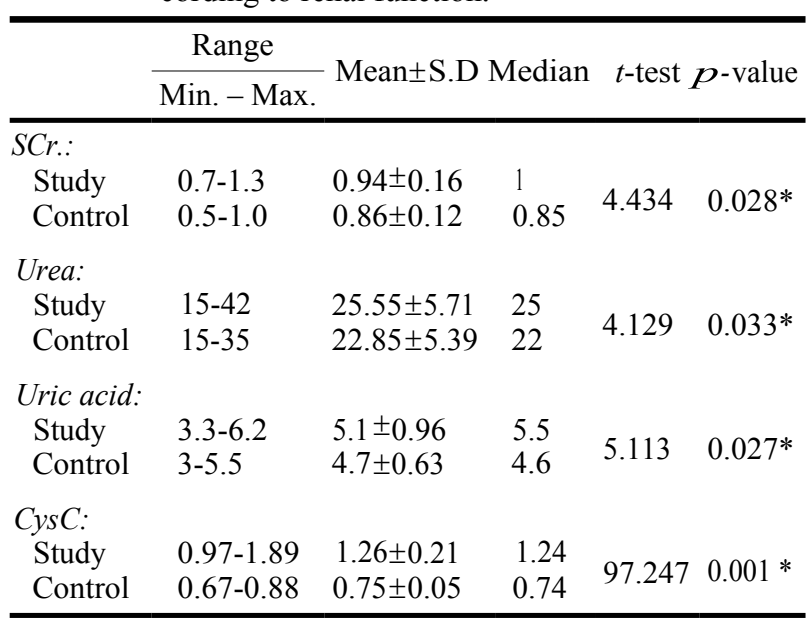

Table (2): Sensitivity, specificity and accuracy for cystatin C, creatinine and uric acid with cases and control group.

\begin{tabular}{lllcccc}
\hline & AUC & $\begin{array}{c}\text { Sensit- } \\
\text { ivity }\end{array}$ & $\begin{array}{c}\text { Specif- } \\
\text { icity }\end{array}$ & PPV NPV & $\begin{array}{c}\text { Accur } \\
\text { acy }\end{array}$ \\
\hline CysC & 0.814 & 96 & 100 & 100 & 95 & 97.5 \\
Uric acid & 0.910 & 87.5 & 100 & 100 & 89 & 94 \\
SCr. & 0.972 & 82.5 & 100 & 100 & 85 & 91 \\
\hline
\end{tabular}

AUC: Area under curve.

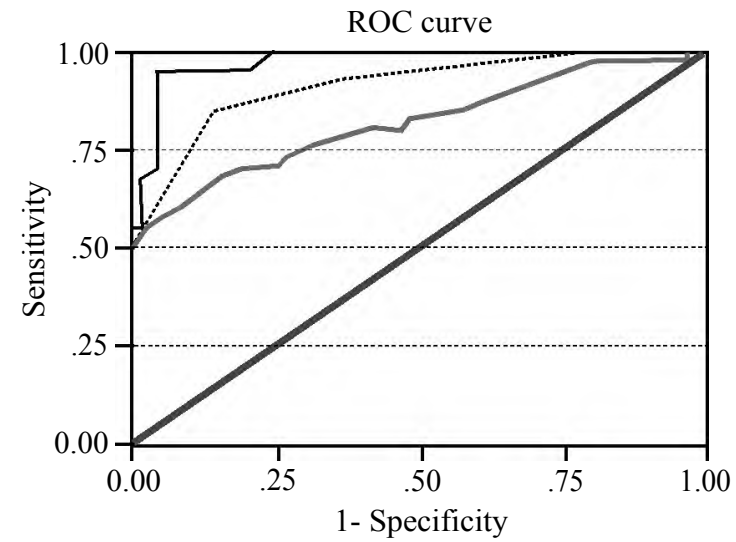

Diagonal segments are produced by ties

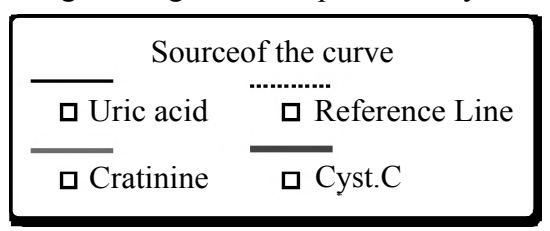

Fig. (1): ROC (receiver operating characteristic only curve) for cystatin $\mathrm{C}$, creatinine and uric acid showing that serum cystatin $C$ has superior diagnostic accuracy than creatinine and serum uric acid and serum uric acid was better than serum creatinine.

\section{Discussion}

Preeclampsia can cause changes in many organ systems, the predominant pathology, which is endothelial dysfunction, starts as early as $8-18$ week but the signs and symptoms appear in the late mid-trimester. One of the most common complications of PE is renal dysfunction [13] . In order to arrest the disease process in early stages and to prevent complications, various predictors have been proposed. BUN and $\mathrm{SCr}$ are commonly elevated parameters in any renal failure. Searching for an ideal renal marker in PE, which may overcome the limitations of the traditional markers, the present study on cysC was done. Markers of kidney function were investigated in the two groups, the difference in the mean serum concentration of creatinine $(0.94 \pm 0.16$ versus $0.86 \pm 0.12 \mathrm{mg} / \mathrm{dl})$ was significantly higher in PE $(p=0.028)$, the difference in the mean blood urea $(25.55 \pm 5.71$ versus 22.85 $\pm 5.39 \mathrm{mg} / \mathrm{dl}$ ) was significantly higher in PE $(p=0.033)$. These data agree with the studies carried out by Singh et al., [14] ., Roberto et al., [15] and Mikic et al., [16]

Serum uric acid levels decrease in first trimester and then increase during pregnancy, with the levels in the third trimester being significantly higher compared to the levels of non-pregnant women. Hyperuricemia is often associated with preeclampsia [17]. In this study, the difference in the mean serum uric acid level $(5.10 \pm 0.96$ versus $4.70 \pm 0.63$ $\mathrm{mg} / \mathrm{dl})$ was significantly higher in PE $(p=0.027)$. Similar results were reported in studies by Singh et al., [14], Sharma et al., [18] and Mikic et al., [16]

Cystatin $\mathrm{C}$ is glomerular filtration rate marker that performs better diagnostically than creatinine. Its main advantage is that it is less dependent on body composition than creatinine [11]. In PE, the serum concentration of cysC relates significantly to structural and functional changes in the kidneys. [12] . In this study, the difference in the mean serum concentrations of cystatin C $(1.26 \pm 0.21$ versus $0.75 \pm 0.05 \mathrm{mg} / \mathrm{l})$ was significantly higher in $\mathrm{PE}$ $(p=0.001)$.

In a cross-sectional study by Singh et al., [14], 75 subjects enrolled, 25 of normal pregnancy, 25 of $\mathrm{GH}$ and 25 of PE. serum cysC, blood urea, $\mathrm{SCr}$ and serum uric acid were estimated in all subjects. They found that all renal parameters including cys C were significantly raised in $\mathrm{GH}$ and $\mathrm{PE}$ compared with control group; However, only serum cysC levels was significantly higher in patients with $\mathrm{PE}$ than $\mathrm{GH}$ and control pregnant females, the mean was $1.62 \pm 0.32 \mathrm{mg} / 1,1.26 \pm 0.28 \mathrm{mg} / 1$ and $0.96 \pm 0.23 \mathrm{mg} / 1$ respectively. Roberto et al., [15] ., made a study on 67 women with PE and 20 normotensive pregnant women matched for gestational age. Levels of blood urea, $\mathrm{SCr}$ and cysC were evaluated. CysC level in PE women was signifi- 
cantly higher than in the normotensive one. In a study by Sharma et al., [18], 60 patients (30 with $\mathrm{PE}$ and 30 healthy pregnant females) were included. Estimation of cysC, $\mathrm{SCr}$, urea, uric acid was made. Serum cysC concentrations were significantly higher in PE patients $(1.31 \pm 0.4 \mathrm{mg} / \mathrm{L})$ compared to the healthy pregnant females $(0.96 \pm 0.2 \mathrm{mg} / \mathrm{L})$ with $p$-value $<0.001$. Similarly, serum uric acid was also significantly higher in PE patients than controls $(p=0.002)$. In a study by Mikic A.N. et al., [16], Markers of kidney function were investigated in two groups of pregnant women: One with $\mathrm{PE}(\mathrm{n}=32)$ and the other of healthy pregnant women $(n=60)$. Serum levels of cysC, creatinine and uric acid were significantly higher in the PE group than in the control group.

In this study receiver operating characteristic analysis of the various renal markers revealed that serum cystatin $\mathrm{C}$ has superior diagnostic accuracy than creatinine and serum uric acid to detect renal dysfunction in PE, for serum uric acid level, the specificity was $100 \%$ and sensitivity was $87.5 \%$, with accuracy $94 \%$. For serum creatinine, the specificity was $100 \%$ and sensitivity was $85 \%$, with accuracy $91 \%$. For serum cystatin $C$, the specificity was $100 \%$ and sensitivity of the test was $96 \%$, with accuracy $97.5 \%$ which were maximum. These finding agrees with Singh A. et al., [14] where cysC had a higher sensitivity (94\%) and specificity $(88 \%)$ than other conventional markers.

We have come across one study by Padma et al., [19], in which levels of serum cysC, $\mathrm{SCr}$ and uric acid were measured in samples from 69 healthy women at term as well as in 27 samples of patients with $\mathrm{GH}$ and in 20 patients with PE. Levels of all three components were significantly higher in PE patients with the mean \pm SD being $1.47 \pm 0.9 \mathrm{vs}$. $1.06 \pm 0.2$ for cysC, $0.95 \pm 0.2$ vs. $0.67 \pm 0.1$ for $\mathrm{SCr}$ and $6.13 \pm 1.8$ vs. $4.28 \pm 1.1$ for uric acid respectively. In $\mathrm{GH}$ cysC was significantly higher, $1.25 \pm 0.9$ unlike $\mathrm{SCr}, 0.67 \pm 0.14$ and uric acid, $4.30 \pm 1.0$. Receiver operating characteristic (ROC) plots demonstrated that the diagnostic accuracy of $\mathrm{SCr}$ was superior to serum uric acid and serum cysC, and serum uric acid was better than serum cysC. One possible reason could be that they have used Jaffe's reagent-based kinetic assay than enzymatic methods used in our study, which has been shown to be more accurate with reference method.

\section{Conclusion:}

Although blood urea, serum creatinine and serum uric acid are good predictors for renal dysfunction in preeclampsia when positively elevated yet normal value doesn't exclude kidney affection.
However, serum cystatin $\mathrm{C}$ is an accurate and early predictor for renal dysfunction in preeclampsia and can detect any renal affection when others (blood urea, serum creatinine and serum uric acid) are normal.

\section{References}

1- American College of Obstetricians and Gynecologists: Hypertension in pregnancy. Report of the American College of Obstetricians and Gynecologists' Task Force on Hypertension in Pregnancy. Obstetrics Gynecology, 122 (5): 1122-31, 2013.

2- CHEUNG K.L. and LAFAYETTE R.A.: Renal Physiology of Pregnancy. Advances in Chronic Kidney Disease, 20 (3): 209-214, 2013.

3- JAMES G.D., SEALEY J.E., ALDERMAN M., LJUNGMAN S. and MUELLER F.B.: A longitudinal study of urinary creatinine and creatinine clearance in normal subjects. Race, sex, and age differences. Am. J. Hypertens, 1: 124-31, 1988.

4- LEVEY A.S., BERG R.L., GASSMAN J.J., HALL P.M and WALKER W.G.: Creatinine filtration, secretion and excretion during progressive renal disease. Kidney Int., 36 (27): S73-80, 1989.

5- PERRONE R.D., MADIAS N.E. and LEVEY A.S.: Serum creatinine as an index of renal function: New insights into old concepts. Clin Chem., 38:1933-53, 1992.

6- ABRAHAMSON M., OLAFSSON I., PALSDOTTIR A., ULVSBACK M., LUNDWALL A., JENSSONT O. and GRUBB A.: Structure and expression of the human cystatin C gene. Biochem. J., 268: 287-294, 1990.

7- OCHIENG J. and CHAUDHURI G.: Cystatin superfamily. Journal of Health Care for the Poor and Underserved, 21 (1): 51-70, 2010.

8- ODUTAYO A. and CHERNEY D.: Cystatin C and acute changes in glomerular filtration rate. Clinical Nephrology, 78 (1): 64-75, 2012.

9- CHANTREL F., AGIN A., OFFNER M., KOEHL C., MOULIN B. and HANNEDOUCHE T.: Comparison of cystatin $\mathrm{C}$ versus creatinine for detection of mild renal failure. Clin. Nephrol., 54: 374-81, 2000.

10- CRODATODD M.T., SOTOMONTANO X.J., HERNÁNDEZCANCINO P.A. and JUÁREZAGUILAR E.: "Adult cystatin $\mathrm{C}$ reference intervals determined by nephelometric immunoassay" Clin. Biochem., 40 (1314): 1084-1087, 2007.

11- KRISTENSEN K., LINDTROM V., SCHMIDT C., BILRUP-JENSEN S., GRUBB A. and WIDE-SWENSSON D.: Temporal changes of the plasma levels of cystatin C, beta-trace protein, beta2-microglobulin, urate and creatinine during pregnancy indicate continuous alterations in the renal filtration process. Scand. J. Clin. Lab. Invest., 67: 612-8, 2007.

12- THILAGANATHAN B., RALPH E., PAPAGEORGHIOU A., MELCHIORRE K. and SHELDON J.: Raised serum cystatin C: An early pregnancy marker for preeclampsia. Reprod Sci., 16: 788-93, 2009. 
13- MORAN P., LINDHEIMER M.D. and DAVISON J.M.: The renal response to preeclampsia. Seminars in Nephrology, 24: 588-595, 2004.

14- SINGH A., GUPTA M., RANJAN R., SAINI V. and GUPTA S.: Cystatin C is a Better Marker of Renal Dysfunctions in Hypertensive Pregnancies. Indian J. Med. Biochem., 20 (1): 21-27, 2016.

15- ROBERTO A.C., MARIA T.P., MARIANA R., INGRID C.W., JOELCIO F.A. and JOSE C.P.: Association between cystatin $\mathrm{C}$ and markers of renal function in preeclamptic pregnant women. Pregnancy Hypertension: An International Journal of Women's Cardiovascular Health, 5 (1): 64, 2015.

16- MIKIC A.N., VELIBOR C.V., NIKOLIC A., MARIC D., BRKIC S. and MITIC G.: Diagnostic value of cystatin C serum levels as alternative marker of renal function in pre-eclampsia. The Journal of Maternal-Fetal \& Neonatal Medicine, 25 (7), 2012.

17- BAINBRIDGE S.A. and ROBERTS J.M.: Uric acid as a pathogenic factor in preeclampsia. Placenta, 29 (1): $67-$ 72, 2008.

18- SHARMA S., RANJAN R., GUPTA M., SINGH A., GUPTA R., PANT L. and SINGH S.: Serum Cystatin C compared with conventional renal function tests: A study in patients with pre-eclampsia. Annals of Pathology and Laboratory Medicine, 1 (2): A6-A9, 2014.

19- PADMA Y., APARNA V., KALPANA B., RITIKA V. and SUDHAKAR P.: Renal markers in normal and hypertensive disorders of pregnancy in Indian women: A pilot study. International Journal of Reproduction, Contraception, Obstetrics and Gynecology, 1 (2): 514-520, 2016.

\section{مستوى سيستاتين ج بمصل الدم لتقييه وظائف الكلى التهم

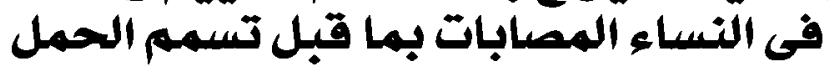

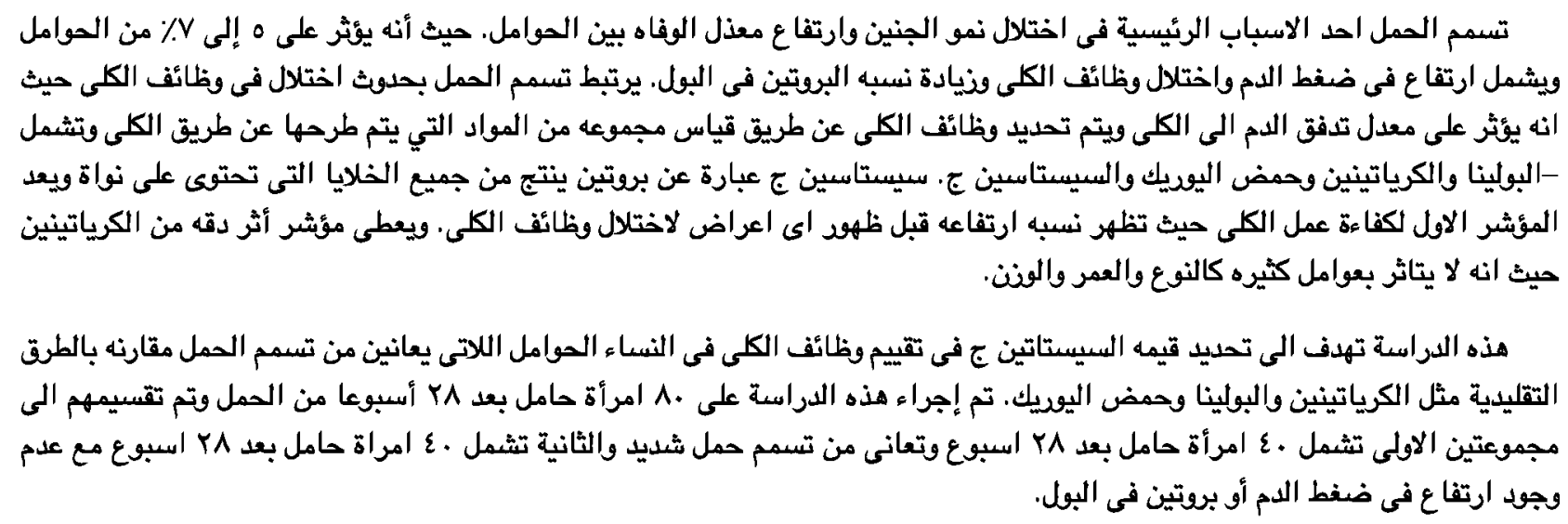

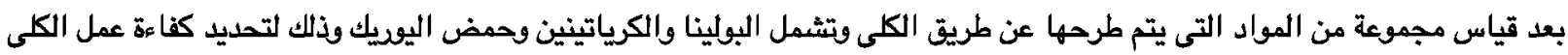

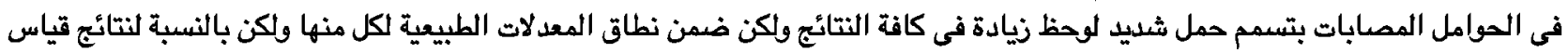

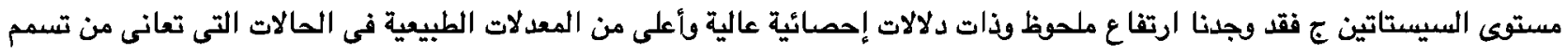

